\title{
Société Neuchâteloise de Médecine
}

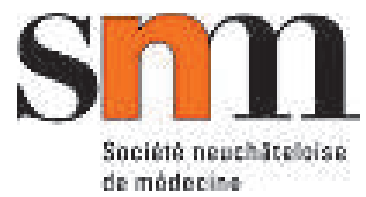

Pierre-Yves Bilat

Präsident SNM

\section{Einleitung}

Die 1852 gegründete Société Neuchâteloise de Médecine (SNM) zählt derzeit 480 Mitglieder aus allen medizinischen Fachgebieten; sie sind sowohl freipraktizierend als auch in Spitälern und anderen Pflegeeinrichtungen des Kantons in leitenden Positionen angestellt.

\section{Gemeinsam in der Vielfalt}

Die Mitglieder der SNM treffen sich zweimal pro Jahr in La Chaux-de-Fonds bzw. Neuenburg zur Generalversammlung. Diese dient nicht nur formellen Zwecken, dem Informationsaustausch und der Beschlussfassung, sondern stellt auch einen beliebten Treffpunkt für Allgemeinpraktiker, Spezialisten, Spitalärzte und freipraktizierende Ärzte sowie Mitglieder aus allen Kantonsteilen dar. In einer überschaubaren Gesellschaft wie der SNM kann man noch jeden kennen. Um das Tagesgeschäft kümmert sich ein Vorstand aus neun Mitgliedern, die die Vielfalt der kantonalen Ärzteschaft vertreten und alle eine besondere Verantwortung wie beispielsweise den Vorsitz einer der ständigen Kommissionen wahrnehmen.

\section{Vom Besonderen zum Allgemeinen}

Wie alle kantonalen Ärztegesellschaften ist auch die SNM praxisnah und beantwortet insbesondere über ihr Rechtssekretariat konkrete Einzelanfragen ihrer Mitglieder im Zusammenhang mit ihrer täglichen Arbeit. Die Informationen innerhalb der Gesellschaft werden ausserdem über das viermal jährlich erscheinende Informationsorgan «SNM News» sowie über die eigene Website (www.snm.ch) verbreitet. Ferner steht den Mitgliedern ein elektronisches Diskussionsforum zur Verfügung.

Die Wissenschaftskommission organisiert äusserst geschätzte halbtägige Weiterbildungsveranstaltungen in den Spitälern und setzt bei der Vorbereitung auf eine Zusammenarbeit mit den freipraktizierenden Ärzten. Dadurch sind diese Anlässe sehr nützlich und praxisorientiert.

Die SNM ist über ihren Präsidenten oder ihre Vorstandsmitglieder direkt im Spitalrat (Conseil des hôpitaux) und im Gesundheitsrat (Conseil de santé) vertreten. Bei letzterem handelt es sich um ein Konsultativorgan des für das Gesundheitswesen zuständigen Regierungsrats. Die Stimme der Ärzte wird also auch auf politischer Ebene gehört.

\section{Sonstige Tätigkeiten}

In wenigen Worten die wichtigsten aktuellen Themen der SNM zu beschreiben, ist ein riskantes Unterfangen: Die Ärztedemographie (in Absprache und Zusammenarbeit mit dem Kantonsarzt), die Einsätze der Notfallärzte auf Ersuchen der Polizei sowie die Beziehungen zwischen den Erstversorgern und dem ambulanten Bereich der Spitäler sind nur einige Beispiele. Beim Verhältnis zu den Versicherern schätzt die SNM die Zusammenarbeit mit dem Regionalbüro von santésuisse zum Thema Wirtschaftlichkeit. Das Vertrauensverhältnis in der paritätischen Kommission SNMsantésuisse hängt zweifellos damit zusammen, dass die SNM während der Periode der Kostenneutralität eine interne «Aufsichtskommission» mit Befugnis zur Untersuchung bei als teuer gemeldeten Ärzten eingerichtet hat. Diese zuerst als «Polizeiorgan» empfundene Kommission stellte ihren Nutzen rasch unter Beweis, weil sie den betroffenen Ärzten half, ihre vom Trust Center gelieferten Zahlen mit den Besonderheiten ihrer Praxis zu begründen und sich in einigen Fällen möglicher Fehler in der Tarifanwendung bewusst zu werden. Seit mehreren Jahren führt im Kanton Neuenburg nur eine winzige Anzahl solcher Fälle zu einem Wirtschaftlichkeitsverfahren. Weniger rosig sieht die Situation jedoch bei den Tarifen aus, weil erstmals seit langem keine Einigung zwischen der SNM und santésuisse über den TARMED-Taxpunktwert 2009 erzielt werden konnte. Folglich ist beim Regierungsrat ein Verfahren zur Festlegung eines Rahmentarifs hängig. Beim Thema Versicherungen ist abschliessend die unter der Ägide der SNM in Gang gesetzte Erarbeitung eines Vertragsentwurfs für ein kantonales Ärztenetzwerk zu nennen. Dass der Hauptversicherungspartner des Projekts plötzlich auf eine Teilnahme am Netzwerk verzichtet hat, ist eine andere Geschichte [1].

\section{Literatur}

1 Tzaut A, Rilliot J. Les médecins neuchâtelois dénoncent l'attitude incompréhensible du Groupe Mutuel. Schweiz Ärztezeitung. 2008;89(43):1848.
Dr. med. Pierre-Yves Bilat

Sekretariat SNM

Grand-Rue 36 bis

CH-2108 Couvet

Tel. 0328632171

Fax 0328631619

www.snm.ch 